\title{
CÓDIGOS DE BARRAS: A PRESENÇA VISÍVEL DA AUTOMAÇÃo
}

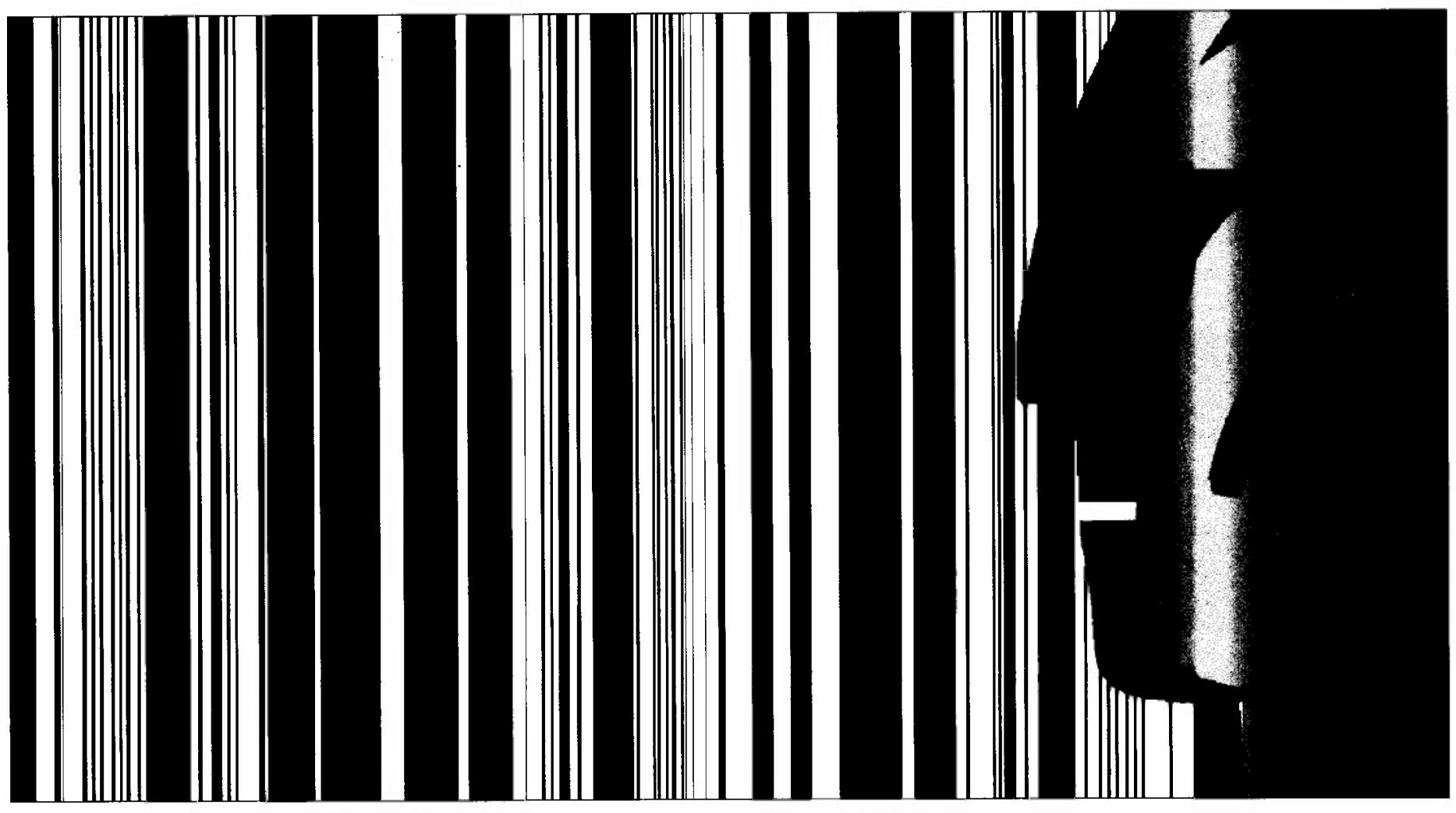

\section{Angelo Soares}

Doutorando em Sociologia na Universidade Laval, Quebec, Canadá, autor do livro O que é informáticaSegunda visão.

\section{INTRODUÇÃO}

automação é um processo irreversível e fundamental A para as sociedades modernas. Trata-se de um de1 safio, principalmente para os países do Terceiro Mundo, pois passamos por um período de reordenação na economia mundial contemporânea, onde novos padrões de concorrência e de qualidade começam a aparecer. Sendo assim, os países do Terceiro Mundo que quiserem manter, ou até mesmo expandir, sua fatia no mercado internacional terão que ter na automação um de seus principais objetivos.

Um dos sinais visíveis da automação é a presença de pequenas barras pretas e brancas, que variam de espessura, existentes nos mais variados tipos de produtos: os códigos de barras (ver figura 1).
Eles estão cada vez mais presentes no nosso dia a dia: seja em produtos nos supermercados, drogarias, correios, em crachás nos aeroportos, nos controles de processos de fabricação. Dessa forma, os códigos de barras afetam nossas vidas, seja enquanto consumidores ou trabalhadores.

Figura 1: Código de Barras

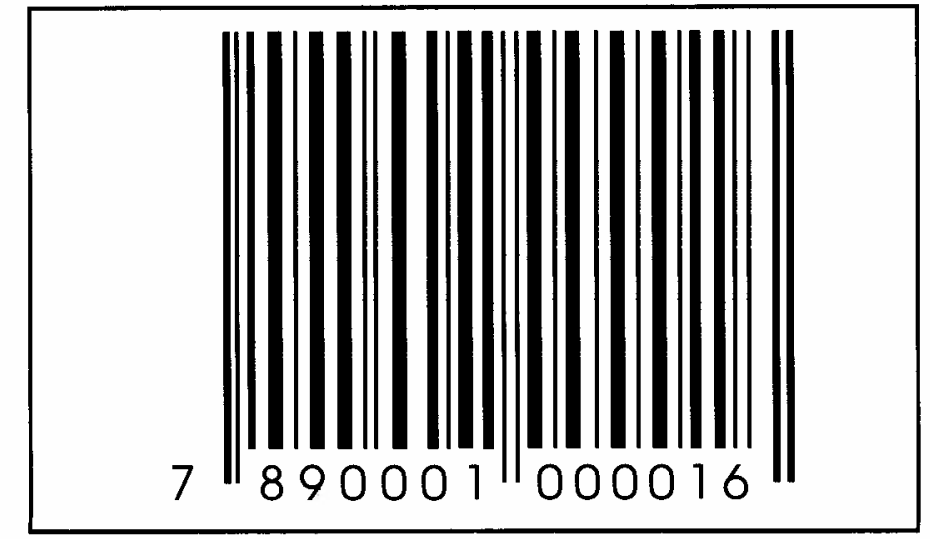




\section{A ENTRADA DE DADOS E OS CÓDICOS DE BARRAS}

Todo sistema computadorizado, para funcionar, requer a introdução de dados que serão processados pelo sistema, que no final desse processo nos fornecerá as informações e/ou serviços desejados. Esquematicamente, podemos resumir os sistemas computadorizados conforme a figura 2 .

Figura 2: Esquema Resumido de um Sistema Computadorizado

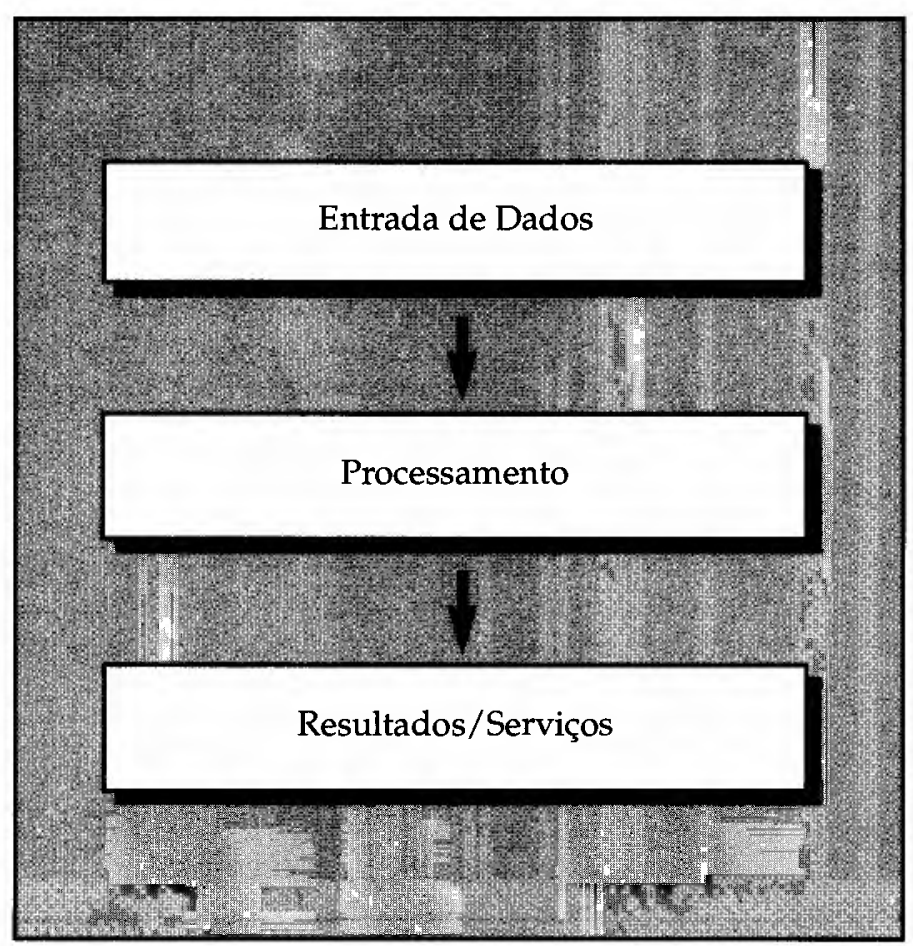

Desde o aparecimento do computador em 1946, o processo de entrada de dados mostrou-se problemático. Trata-se de uma tarefa monótona e repetitiva de baixa qualificação e de baixa remuneração: a perfuração de cartões. Segundo Brabb ${ }^{1}$, o computador permanecia mais de $90 \%$ do seu tempo aguardando as entradas de dados e as instruções para o seu funcionamento.

A partir de 1960, com a Segunda Fase ${ }^{2}$ dos computadores, houve uma redução substancial no tempo de processamento - que passou de milissegundos para microssegundos - e do custo da máquina, o que gerou uma diminuição no valor dos produtos/serviços produzidos. Desse modo, acentuou-se uma contradição: velocidade de processamento dos dados elevada $x$ entrada de dados artesanal, no ritmo de trabalho do(a) operador(a). Segundo Manacorda, tornou-se necessário substituir o(a) operador(a) "por algo mais adaptado à velocidade da máquina, mais rigidamente determinado e menos variável com os ritmos humanos" ${ }^{3}$.

$\mathrm{Na}$ busca da solução dessa contradição e com o crescimento do volume de informações, apareceram os terminais de vídeo já na Terceira Fase (1964-1972) de Computadores, como um novo periférico para entrada de dados.
Entretanto, assim como com os cartões perfurados, os terminais de vídeo mantêm a necessidade de se fazer a crítica da informação transcrita, pois há um percentual elevado de erros (aproximadamente $40 \%$ ), provenientes de vários fatores: falta de ergonomia dos equipamentos e do local de trabalho, sistemas mal desenhados, e falhas humanas. Essas informações erradas, obviamente, não podem ser fornecidas ao computador. Esse é um fator adicional que diminui a velocidade na entrada de dados e que contribui para a sua transformação num "gargalo", que limita sobremaneira o processamento dos dados. A velocidade de processamento, que nessa fase já era um milhão de vezes maior que na dos primeiros computadores, se manteve muito superior ao ritmo de trabalho do(a) operador(a).

A seguir, apareceram novas tecnologias para a entrada de dados: reconhecimento ótico de caracteres (OCR - optical character recognition), bandas magnéticas (presentes nos cartões magnéticos de bancos, por exemplo), processamento de imagens, reconhecimento de vozes e os códigos de barras.

Comparados com a entrada de dados via teclados, os códigos de barras representam um enorme ganho de velocidade no processo, pois, além de se conseguir ler as informações mais rapidamente, o tempo gasto na crítica da informação transcrita foi eliminado, dado que o próprio computador faz a conferência da leitura (por exemplo, através de dígitos verificadores) e a sua taxa de erro é muito menor. Segundo Baker, na entrada de dados via teclados, "a probabilidade de erro quando trabalhamos com 4 dígitos é por volta de 1 em 1000. Em contrapartida, com um código de barras, essa taxa é de 1 erro a cada 3.379 .000 caracteres"

O custo dessas novas tecnologias de entrada de dados também coloca os códigos de barras numa situação privilegiada, pois é o mais barato dentre todas elas. Além disso, eles possuem acurácia e versatilidade muito maiores, por exemplo, quando comparados com as bandas magnéticas, que necessitam de uma leitora que, muitas vezes, limita muito a flexibilidade de sua utilização.

O processo de reconhecimento de voz é muito limitado e possui inúmeras fontes de erros (pronúncia, inflexão, dialetos); além disso, cada palavra deve ser armazenada na memória do computador e será checada toda vez que for pronunciada. Há ainda as interferências, ruídos de fundo que dificultam o reconhecimento da voz e que também são fontes de erros.

Sendo assim, concordamos com Baker ${ }^{5}$ que os códigos

\section{BRABB, G. J. Business Data Processing. Boston, Houghton, 1982.}

2. Não usamos o conceito de "geração" para designar cada uma das fases de desenvolvimento dos computadores, embora seja a nomenclatura mais usual, pois a utilização dessa metáfora acaba nos induzindo a acreditar ou considerar o computador como uma "espécie biológica" que atravessa gerações como se estivesse evoluindo de maneira autônoma, rumo a uma "categoria especial" de uma máquina inteligente, como nos mostra ROSZACK, T. O Culto da Informação. São Paulo, Ed. Brasiliense, 1988.

3. MANACORDA, P. El Ordenador del Capital - Razón y Mito de la Informatica. Madri, H. Blume Ediciones, 1982, p. 91.

4. BAKER, E. F. Industry Shows its Stripes - A new Role for Bar Coding. Nova York, AMA Publishing, 1985, p. 14.

5. Idem, ibidem. 


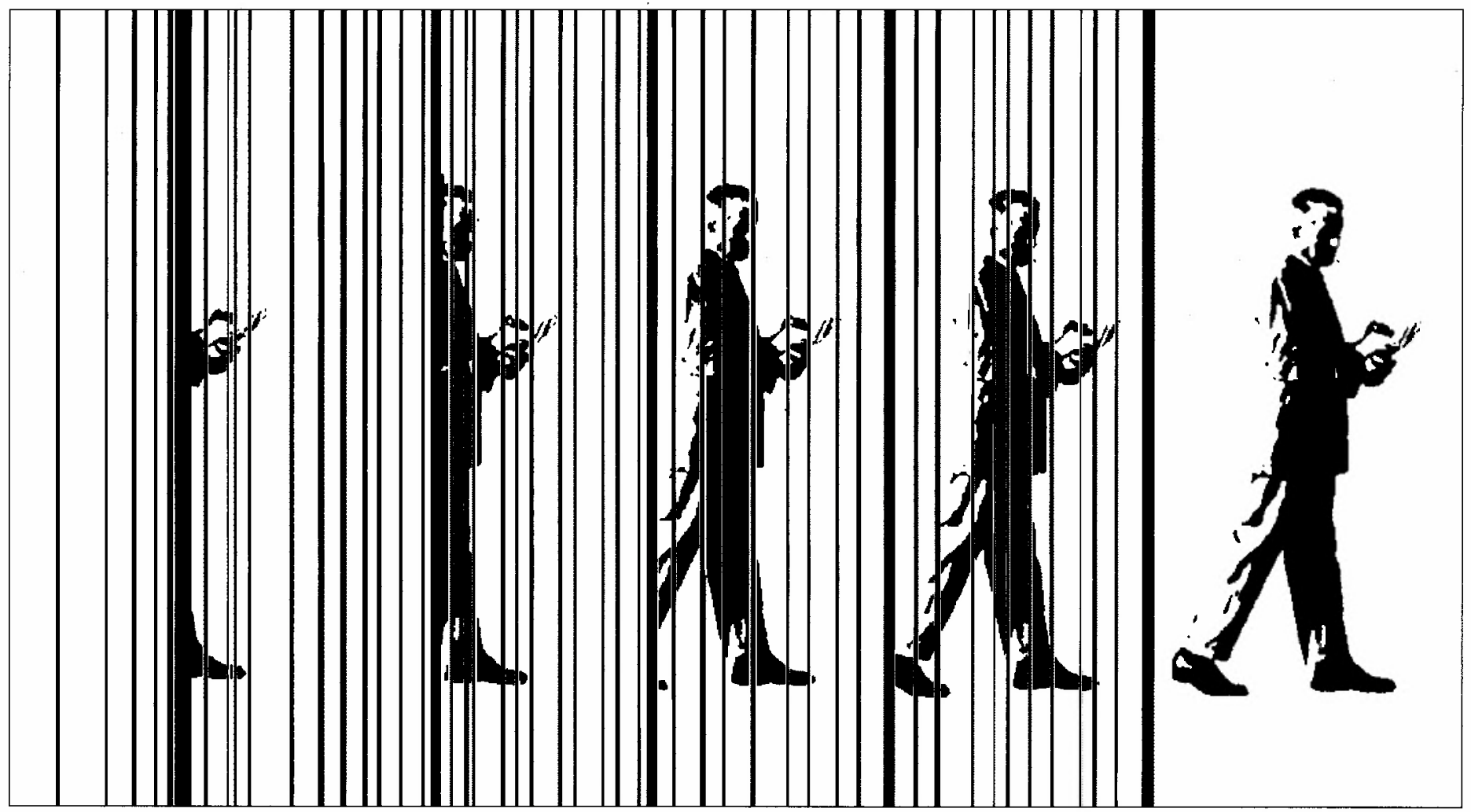

de barras representam a opção de entrada de dados mais produtiva, com maior precisão, melhor taxa de retorno de investimento (nos EUA, de 9 a 18 meses), maior flexibilidade e facilidade no uso.

\section{O QUE SÃo OS CóDICOS DE BARRAS?}

Os códigos de barras são basicamente um conjunto variável de barras paralelas que representam uma determinada informação. A primeira e a última barra representam o início e o final do código respectivamente. Os códigos de barras operam através do princípio de refletividade da luz. O dispositivo de leitura de um código de barras nada mais faz do que emitir um foco de luz que, ao atingir o código, vai refletir as barras claras, que são transformadas em sinais elétricos passíveis de serem convertidos na linguagem binária dos computadores.

Dessa forma, podemos concluir que um dos problemas existentes com os códigos de barras é a presença de manchas, sujeira ou qualquer tipo de deterioração das barras, que provocará uma interrupção na leitura.

Existem vários dispositivos de leitura de códigos de barras: scanners, canetas óticas, pistolas a laser, dentre outros. Baker ${ }^{6}$ aponta alguns aspectos que devem ser considerados ao se definir e/ou escolher um dispositivo de leitura de códigos de barras, por exemplo: o grau de limpeza a que os códigos de barras estarão expostos; a compatibilidade com o sistema de computação utilizado; facilidade no uso; possibilidade de leitura de várias simbologias e freqüência de uso.
O primeiro código de barras desenvolvido foi o 2 de 5 , ainda nos anos 60 , e tem sido utilizado em depósitos de mercadorias e passagens aéreas. Cada caractere é formado por cinco barras, das quais duas são mais largas (daí vem o nome do código). Os espaços em branco são utilizados apenas para separação das barras. Esse código possui alguns problemas: não existem padrões para representar mais de dez dígitos, os códigos se tornam muito longos, o que resulta em dificuldades na leitura com scanners a laser.

Da tentativa de se fazer um código mais compacto a partir do código 2 de 5, em 1972 apareceu o código 2 de 5 intercalado, que é $40 \%$ menor que o anterior. Entretanto, esse código ainda possui uma desvantagem: a de só codificar números e não caracteres.

Em 1975, foi criado o código 3 de 9, onde cada caractere é formado por 9 elementos (5 barras e 4 espaços), dos quais 3 devem ser mais largos. Esse código é bastante utilizado nas indústrias em diversos estágios do processo produtivo, em bibliotecas, serviços hospitalares etc.

Em contrapartida, os códigos utilizados na automação comercial são o UPC-Universal Product Code e o EAN-International Article Number - que pode ser o $E A N-13$,composto de 13 dígitos ou o $E A N-8$, composto de 8 dígitos. $O$ UPC é utilizado apenas nos EUA e Canadá: é composto por 12 dígitos, onde cada caractere é formado por duas barras escuras e duas claras.

6. Idem, ibidem. 
O código EAN é o mais difundido e aceito internacionalmente, inclusive no Brasil (ver figura 3). Sua estrutura é a seguinte:

Identificação do País: Os primeiros dois ou três dígitos da esquerda para a direita, conforme necessidade do país. No caso do Brasil, esse número é o 789.

Identificação do Fabricante e do Produto: Os nove ou dez dígitos subseqüentes ao código do país. Esse código é determinado, no Brasil, pela ABAC - Associação Brasileira de Automação Comercial.

Dígito Verificador: Um dígito que serve de controle para se certificar de que não houve erro na leitura do código. É calculado segundo um algorítmo em 5 passos?.

\section{Figura 3: Código EAN-13}

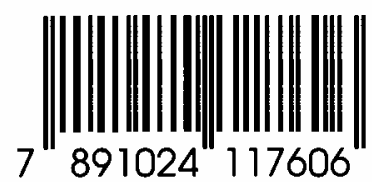

\section{9 - Código do País: Brasil \\ 1024 - Código do Fabricante \\ 11760 - Código do Produto \\ 6 - Dígito de Controle}

Algumas vantagens da utilização do código EAN para a automação comercial são o seu uso na maioria dos países e a possibilidade de utilizar o padrão $E A N-8$ para embalagens menores.

\section{A AUTOMAÇÃO E OS CÓDIGOS DE BARRAS}

Ao contrário do que se possa imaginar, os códigos de barras encontram hoje inúmeras aplicações em diversos setores que estão se automatizando e não só no comércio.

Um aspecto que merece ser destacado é que os códigos de barras têm proporcionado um grande impulso ao desenvolvimento e utilização de sistemas automatizados, em virtude da grande simplificação associada à alta velocidade que eles introduzem na entrada de dados. Entretanto, deve ficar claro que os códigos de barras são apenas uma parte do processo automatizado: a entrada dos dados.

No comércio, a utilização de códigos de barras tem sido intensa e crescente, proporcionando aumentos na produtividade, redução nos custos de colocação de preços nas mercadorias, redução de estoques, e todo o controle da parte contábil automatizado. Segundo Tereesko ${ }^{8}$, uma loja que possua um volume anual de vendas de US\$ $5.2 \mathrm{mi}-$ lhões pode economizar US $\$ 60.000$ com códigos de barras.
Outro aspecto importante no uso dos códigos de barras no comércio é a satisfação do cliente, que tem seu tempo de espera em filas diminuído. Segundo a ABAC - Associação Brasileira de Automação Comercial - um cliente leva 40 segundos para passar com 12 volumes em um caixa que utiliza a leitura de códigos de barras.

$O$ controle de estoques ganha uma agilidade e importância nunca antes existente no comércio: pode-se saber, minuto a minuto, o que se vendeu, a quantidade vendida, a quantidade que resta no estoque de cada item, pode-se saber quais são os produtos mais e menos vendidos etc. Dessa forma, novas estratégias de marketing podem ser desenvolvidas, por exemplo, o Data Base Marketing, que uma grande rede de supermercados procura implementar aqui no Brasil. Trata-se de um sistema que busca individualizar o tratamento dado ao cliente a partir da construção de um banco de dados onde estarão registradas as suas preferências. Assim, "toda vez que um produto novo for lançado, ou estiver em promoção, poderemos (...) avisar o cliente através de uma mala direta. Desse modo, nossa comunicação com ele deixará de ser genérica para ser específica, o que deve melhorar as vendas"

Entretanto, alguns aspectos negativos também estão relacionados ao uso de códigos de barras no comércio. Num primeiro momento, a introdução dos códigos de barras impõe uma mudança na cultura do consumidor, que passa a não ter mais o preço marcado no produto e sim um código. O preço, agora, está fixado nas gôndolas ou em painéis e o consumidor necessita procurá-los, o que nem sempre é uma atividade fácil. Muitas vezes, o preço sequer está fixado e aí se deve recorrer à operadora de caixa para que ela faça uma consulta.

Esse aspecto negativo parece ser passageiro e só surge no momento da transição para os códigos de barras.

Outro aspecto negativo é a relativa fragilidade dos códigos de barras, que estão freqüentemente sujeitos a condições adversas, por exemplo, em produtos congelados e/ou refrigerados, manchas ou rupturas por contato que deterioram os códigos e dificultam sua leitura. Nesses casos, geralmente, o(a) operador(a) deve introduzir a informação manualmente.

$\mathrm{Na}$ indústria, a utilização de novas técnicas de produção baseadas em "estoques zero", especialização flexível, Just-in-Time CIM - Computer Integrated Manufacturing - necessita de entradas de dados e gerenciamento de informações rápidos e confiáveis. Aqui, novamente, os códigos de barras são extremamente úteis e eficientes. Em alguns casos, sua não utilização inviabiliza o próprio emprego desses novos sistemas de produção.

Na indústria, os códigos de barras podem ser usados em diversos setores: controle de estoques, acompanhamento de pedidos, planejamento e acompanhamento da produção, compra e recebimento de materiais, movi-

7. SILVA, V. L. P. Aplicações Práticas do Código de Barras. São Paulo, Ed. Nobel, 1989.

8. TEREESKO, J. "Why the Boom?" Food's Success Leads Manufacturing". Industry Week, 224, fevereiro 18, 1985.

9. "COMÉRCIO à Luz do Laser". Exame Informática, 08/08/90, p.16-7. 
mentação de materiais, manutenção, controle de qualidade.

Pode-se acompanhar, através da utilização de códigos de barras, cada etapa do processo produtivo on-line, o que nos permite saber o que está acontecendo a cada momento na linha de produção. Dessa forma, se estivermos acompanhando o processo de fabricação de um determinado produto e observarmos o aparecimento de um determinado problema, podemos corrigi-lo quase imediatamente, o que nos proporciona grande economia de tempo e matéria-prima e melhoria na qualidade do produto.

No setor de serviços também é grande a utilização de códigos de barras: em hospitais, para o controle de exames de laboratórios; nas escolas, editoras, livrarias. Um setor que utiliza códigos de barras são as bibliotecas. A cada usuário é fornecido um código de barras, onde estão seus dados pessoais (nome, endereço, número de inscrição na biblioteca etc.) e em cada livro está presente um código de barras que possui as informações referentes ao livro (título, autor, editora etc.). Quando se deseja retirar o livro da biblioteca, basta ler o código do usuário e do livro, e o sistema faz o controle dos dias de empréstimo, multa por atraso etc. Pode-se saber, nesse sistema, onde está cada livro sem a utilização de fichas que podem se extraviar, rasgar ou ser preenchidas de maneira incorreta.

Em alguns aeroportos, encontramos códigos de barras na identificação do destino das bagagens.

\section{OS CÓDIGOS DE BARRAS NO BRASIL}

A utilização de códigos de barras no Brasil tem sido crescente nos últimos anos, principalmente no comércio, em inúmeras lojas, farmácias e supermercados. Um dos pontos que tem dificultado a implementação e utilização dos códigos de barras é a sua baixa taxa de utilização. Apenas $15 \%$ dos produtos estão codificados. Na verdade, existe um certo círculo vicioso: não se codificam os produtos porque a utilização de scanners, leitoras óticas etc. ainda é muito incipiente no país. Por outro lado, as empresas e supermercados não conseguem automatizar suas lojas, pois os produtos não estão codificados.

Entretanto, isso não foi motivo para que grandes redes de supermercados decidissem se automatizar. Para isso, entretanto, o supermercado necessita colocar etiquetas com códigos de barras nos produtos que ainda não estão codificados.

Uma saída para esse problema é adoção de medidas que foram tomadas em países desenvolvidos, por exemplo, a supressão dos fornecedores que não codificarem seus produtos, ou ainda, a cobrança dos custos da codificação feita no supermercado do fornecedor.

No quadro 1, podemos observar a evolução da codificação dos produtos por ramo comercial, que permite sermos otimistas com relação à crescente implementação e utilização dos códigos de barras no nosso país.

\section{Quadro 1: Evolução da Codificação no Brasil}

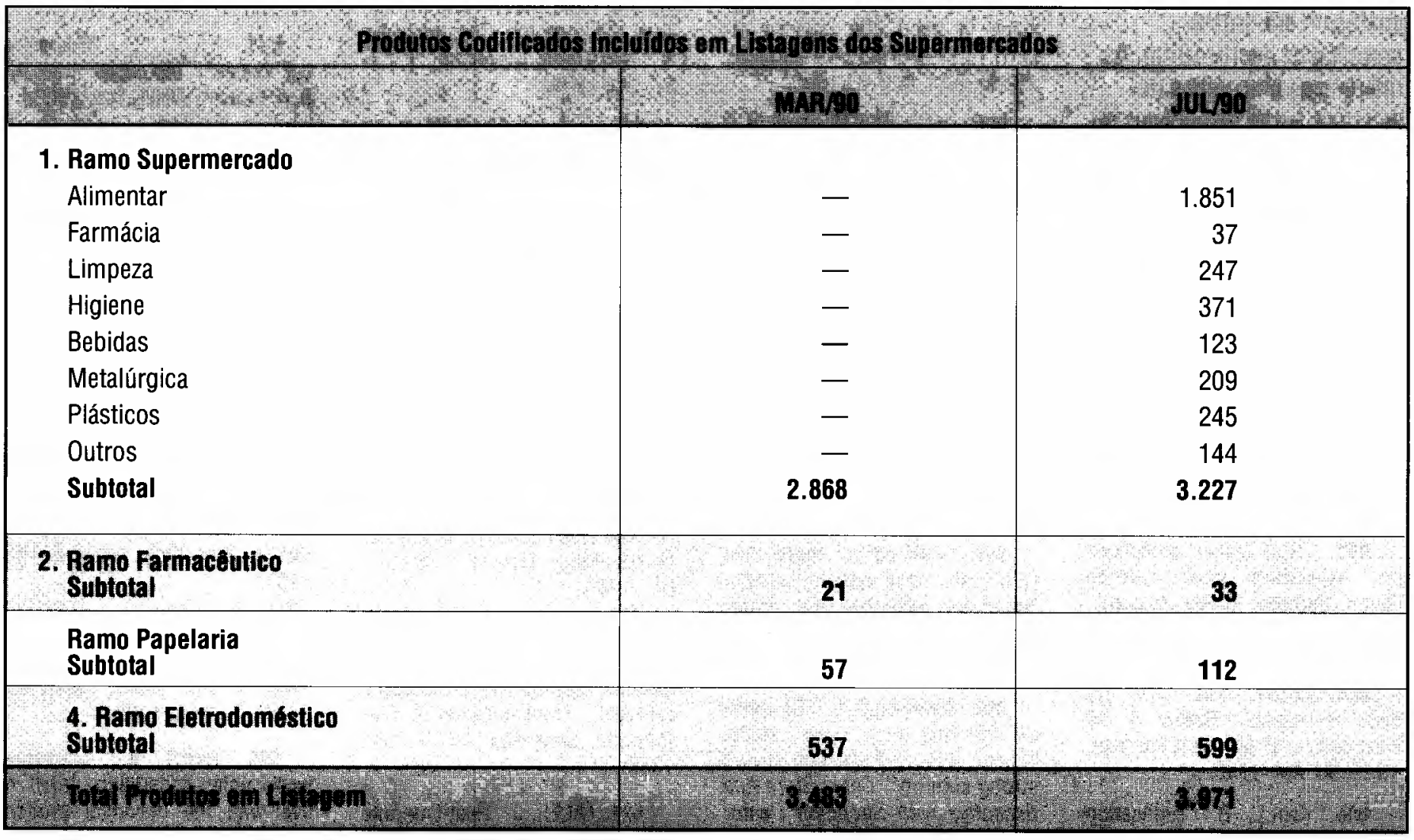

Fonte: Nielsen Serviços de Marketing in Superhiper, out/1990. 
$\mathrm{Na}$ indústria e no setor de serviços, a utilização de códigos de barras é ainda muito incipiente, quando comparada à realidade dos países desenvolvidos. Entretanto, assim como no comércio, à medida que os empresários começarem a descobrir as potencialidades dos códigos de barras na busca da eficiência, produtividade e qualidade, que serão variáveis fundamentais e decisivas, principalmente em virtude da política industrial que se esboça num futuro próximo, os códigos de barras entrarão maciçamente em todos os setores da nossa economia ${ }^{10}$.

10. Agradeço a Denise Mallette e a Jacques Blain do IRSST - Institut de Recherche en Santé et en Securité du Travail du Québec - pelo envio de artigos sobre códigos de barras não disponíveis nas bibliotecas brasileiras.

\section{REFERÊNCIAS BIBLIOGRÁFICAS}

Heraldo Vasconcellos - Bibliotecário, chefe do Serviço de Documentação da Biblioteca Karl A. Boedecker da EAESP/FGV.

\section{OBSERVAÇŌES}

A pesquisa foi realizada no acervo da Biblioteca da EAESP/FGV; as referências bibliográficas foram organizadas em ordem alfabética, dentro de cada ano de publicação (os anos aparecem em ordem cronológica decrescente). As referências precedidas de asterisco estão disponiveis no acervo da Biblioteca.

\section{0}

001. *AUTOMAÇÃO comercial: um investimento que produz eficiência". Superhiper, São Paulo, 16(10):54-6+, out. 1990.

002. "BAR code data collection is the key in the success of NCR's JIT processes". Industrial Engineering, Atlanta, 22(9):30-1, Sept. 1990.

003. "BISG issues guidelines for bar-coding book cartons (Book Industry Study Group)". Publishers Weekly, Riverton, 237:19, Jan. 121990.

004. KIRBY, R.M. "The bar code revolution". Fueloil \& Oil Heat and Solar Systems, Fairfield, 49.29-30, Apr. 1990.

005. KNILL, B. "Unification stimulates technology use (EEC promoting bar coding and EDI)".
Transportation \& Distribution, Cleveland, 31: 30-2, Feb. 1990.

006. "NEW developments in bar coding (distributors)". Industrial Distribution, Denver, 79.106, Jan. 1990

\section{9}

007. "AUTO, ID \& EDI: managing in the "90s (special report)". Industry Week, Cleveland, 238:A1-A28, Aug. 211989.

008. "AUTOMATIC identification buyers guide". Distribution, Radnor, 88:94-5, June 1989.

009. "BAR code system insures accurate inventory control (Simpson Timber Co.)". Forest Industries, San Francisco, 116.26-7, Sept. 1989.

010. "BAR-coded lumber arrives: pergament introduces preaffixed UPC stickers". Chain Store Age Executive with Shopping Center Age, New York, 65:92-4, Aug. 1989.

011. "BAR-codes: more than UPC in the retail industry". Chain Store Age Executive with Shopping Center Age, New York, 65:206-7, Nov. 1989.

012. "BAR-coding, EDI interest running high, says study: Touche Ross/ NRMA poll shows strong support". Chain Store Age Executive with Shopping Center Age, New York, 65:98+, Mar. 1989.
013. "BAR-coding standards". Traffic Management, Denver, 28:61, 0ct. 1989.

014. COCHRAN, T.N. "Quick read (R. Chesek's favorite stock: Symbol Technologies)". Barron's, Chicopee, 69:66, Oct. 21989.

015. CLANCY, D.A. \& IHLENFELD, D.G. "Auto ID and EDI go hand-in-hand (Scan-Tech 89, San Jose, California)". Transportation \& Distribution, Cleveland, 30.578+, Oct. 1989

016. COOKE, J.A. "Auto ID systems gain in popularity". Traffic Management, Denver, 28:16, Feb. 1989.

017 How to pick a bar-code system". Traffic Management, Denver, 28:55-7+, Oct. 1989.

018. DAVIS, L. "Winder uses for bar codes". Nation's Business, Washington, 77:34+, Mar. 1989

019. “DISTRIBUTORS moving toward EDI and bar coding (touche Ross Study)". Industrial Distribution, Denver, 78:13-4, Oct. 1989.

020. DULANEY, T.C. "Interest in bar codes keeps growing (distribution and transportation applications)". Transportation \& Distribution, Cleveland, 30.50, Mar. 1989.

021. FALES, J. "Real-time info is key to profitability (low-error data collection)". Transportation \&
Distribution, Cleveland, 30. 45-6, Sept. 1989.

022. FITZELL, P. "Whole lotta codin' goin on (InterBev, show, Las Vegas)". Beverage World, Great Neck, 108:60+, Dec. 1989.

023. FODOR, G.M. "Bar coding ranks as top topic for 1989". Industrial Distribution, Denver, 78:51, Dec. 1989.

024. "Designing bar codes". Industrial Distribution, Denver, 78:61, Feb. 1989.

025 "Forms printers do double-duty (printing labels with bar code symbols)". Industrial Distribution, Denver, 78:119, Nov. 1989.

026. GROSS, A.E. "Curring the misredemption malady". $A d-$ week's Marketing Week, New York, 30:32-3, May 11989 (supp. Promote)

027. JOHNSTON, P. "What' s new with bar codes?" Graphic Ants Monthly and the Printing Industry, New York, 61:61-2, Feb 1989.

028. JONES, V.A. "Advances continue in micrographics techniques". The Office, Stamford, 110:80-1, Sept. 1989.

029. KERR, K. "Marketing week pole: consumers learn to live with the universal product code". Adweek's Marketing Week New York, 30:22, Sept. 181989. 
030. LAMOURIE, M. "WIM: weigh-in motion devices; $A C I$ automatic container identification (cargo weighing system for ferry ships)". American Shipper, Jacksonville, 31:58+, Feb. 1989.

031. LONGE, K. "Bar coding permeates the health carefield (Health Industry Bar Code - $\mathrm{HI}$ BC - Supplier Labeling Standard)". Hospitals, Chicago, 63:FB56+, July 51989.

032. "MAKING automatic ID pay off (automobile parts)". Automotive News, Detroit:E8+, Dec. 111989.

033. MUTTER, J. "Bookland EAN comes to the book world". Publishers Weekly, Marion, 235:441-4, Jan. 271989.

034. *PINHEIRO, Vera Lúcia. Aplicações práticas de código de barras. São Paulo, Nobel, 1989. $90 \mathrm{p}$.

035. ROBINS, G. "New role for the buyer (retailers)". Stores, New York, 71:34-7, Dec. 1989.

036. "Using the UPC catalog". Stores, New York, 71:61-4, Feb. 1989.

037. ROUGHTON, J. "Scanning the lines for security (bar code data collection system)". Security Management, Arlington, 33:S1-2, Jan. 1989.

038. SADOWSKI, $C$. et alii. "Here's how to use a bar code system to manage lab information". Research \& Development, Denver, 31:38-43, Mar. 211989.

039. SEYMOUR, W. "Bar codes and configurations (montage banking)". Mortgage Banking, Washington, 49:33-6+, Feb. 1989.

040. SPARKS, M. "Data entry with the wave of a wand (bar code data collection system, Riverside Country Publishing)". American Printer, Chicago, 203:S2-3, June 1989

041. STEC, S.F. "NAA presents bar code update (National Association of Accountants)". Management Accounting, New York, 70.54, Apr. 1989.

042. STELTZER, T.M. "Auto ID: where we're headed". Distri- bution, Radnor, 88:93-5, June 1989.

043. "STREAMLINING the logistics pipeline (innovations in order entry, bar coding etc.)". Distribution, Radnor, 88:42+, Dec. 1989.

044. "TRADITION meets technology (Jack Daniel Distillery installs Videojet coding equipment)". Beverage World, Great Neck, 108:189-90, Oct. 1989.

045. TRUNICK, P.A. "Keep an eye an your freight (shipment tracking)". Transportation \& Distribution, Cleveland, 30:20-3, June 1989

046. "VENDORS slow to implement Quick Response: VICS study shows litlle appreciation of cost benefits (voluntary Interindustry Communications Standards)". Chain Store Age Executive with Shopping Center Age, New York, 65:107+, Jan. 1989.

047. WARD, R.V. \& BLISS W.L. "A broad brush approach to bar codes (access control bar code system)". Security Management, Arlington, 33:52t, Jan. 1989 .

048. WESTON, J.M. "Information integration through bar coding". Appliance Manufacturer, Solon, 37:74, Ago. 1989.

049. WILLIAMS, T.C. "As casy as $A B C$ (to sequence letter mail)" Direct Marketing, Garden City, 52:23, June 1989.

050. ZAIGER, P. "Computer tracking network helps improve mill's inventory control (Hollingsworth \& Vose)". Pulp \& Paper, San Francisco, 63:150-1, Apr. 1989.

\section{8}

051. ABEND, J. "Q.R.: changing the D.C. (Quick response)". Stores, New York, 70.89-91t, Apr. 1988.

052. "AS VENDAS eletrônicas". Problemas Brasileiros, São Paulo, 25 (266):4-7, mar./abr. 1988.

053. ASSOCIAÇÃO BRASILEIRA DE AUTOMAÇÃO COMER-
CIAL. Anuário $A B A C$ 1988. São Paulo, 1988. 45p.

054. "AT J.C. Penney, lowly ribbons containing costs". Transportation \& Distribution, Cleveland, 29:74+, May 1988.

055. * A AUTOMAÇÃO chega ao comércio". Exame Informática, São Paulo, 10.3-5, out. 1988.

056. "AUTOMATIC ID taking giant step forward". Transportation \& Distribution, Cleveland, 29.29-30, Oct. 1988.

057. "AUTOMATIC identification buyer's guide (table)". Distribution, Radnor, 87:106-7, Sept. 1988.

058. "AUTOMATIC identification suppliers guide". Transportation \& Distribution, Cleveland, 29.30+, Oct. 1988.

059. "BAR-code scanner solves distribution dilemma". Traffic Management, Denver, 27:69, Jan. 1988.

060. "BAR code symbology: necessary for fashion goods?" Stores, New York, 70.56, Aug. 1988.

061." "BAR coding terms". Management Accounting, Montvale, 69(10): 42-3, Apr. 1988.

062. "BAR codes by 1995 is PMG Frank's aim (Postmaster General T. Frank)". Direct Marketing, Garden City, 51:22+, Nov. 1988.

063. CLIFTON, R.G. "Codes and symbols". Industrial Distribution, Denver, 77:138, Mar. 1988.

064 "De-mystifying bar codes". Industrial Distribution, Denver, 77:94, Sept. 1988.

065. DULANEY, T.C. "Bar-code operations popping up all over". Transportation \& Distribution, Cleveland, 29.55, Mar. 1988.

066. ETTORRE, J.J. "Auto ID is finding motic uses". Transportation \& Distribution, Cleveland, 29.22-3, July 1988.

067. EVANS-CORREIA, K "Signed, sealed, delivered (using bar coding to sort mail)". Purchasing, Denver, 105:98-9, Nov. 241988.
068. "GOAL for PLG: pre-ticketing with UPC". Stores, New York 70. 30-1, Mar. 1988.

069. *GRAHAM, J. "Bar codes becoming universal". Advertising Age, Chicago, 59(17):36, Apr. 18 1988.

070. FITZELL, P. "Breaking the code" (Inter Bev show, Atlanta). Beverage World, Great Neck 107:90-1, Dec. 1988.

071. FODOR, G.M. "Wedging bar codes into PC's". Industrial Distribution, Denver, 77:97, Nov. 1988.

072. HARRINGTON, L.H. "Bar codes booming, new study shows". Traffic Management, Denver, 27:18, 1988.

073. *HICKS, Peter. "Um exército com novo armamento". Superhiper, São Paulo, 14(9):172-7, set. 1988

074. KRAKER, J. "Materials managers change their stripes". ENR, Hightstown, 220.18-9, Mar. 241988.

075. LEFER, H. "Emery focuses on optics for shipment tracking, billing (programmable, handheld bar code reader/computer)". Air Transport World, Cleveland 25:86, Nov. 1988.

076. MENDES, Luis et alii. "Comércio abre suas portas à automação". PC Mundo, Rio de Janeiro, 4(31):14-26, Fev. 1988.

077. MÍDIA INFORMÁTICA. Elementos de um sistema de códigos de barras. São Paulo, 1988. 89p.

078. PAXTON, J.W. "Intricate strategic alliances". Transportation \& Distribution, Cleveland, 29.32-3+, Dec. 1988

079. POLAKOFF, J.C. "Bar coding: realizing an untapped productivity potential". The CPA Journal, New York, 58:98-9, Jan. 1988.

080. "READER'S digest adopts bar coding". Graphic Arts Monthly and the Printing Industry, New York, 60.94t, Sept. 1988.

081. ROBINS, G. "EDI and the buyer: less grunt work". Stores, New York, 70.112-14, May 1988. 
082. "How UPC at point-of-sale is speeding up transactions for faster checkouts (universal product code)". Stores, New York, 70.65-6, Dec. 1988.

083. SCHWEDELSON, $R$. "New wave database". Direct Marketing, Garden City, 50.40-1+, Mar. 1988.

084. * STEC, S.F. "Manufacturing control through bar coding at target products". Management Accounting, New York, 69(10):47, Apr. 1988.

085. "THERE'S nothing horrible about Haggar's network". Transportation \& Distribution, Cleveland, 29.66+, Jan. 1988

086. *TYSON, T. \& SADHWANI, A.T. "Bar codes speed factory floor reporting". Management Accounting, New York, 69(10):41-3+, Apr. 1988.

087. VERTROOST, D. "Bar code standards going international". Transportation \& Distribution, Cleveland, 29.22-4, Oct. 1988.

088. YANACEK, F. "Working inventory wonders". Transportation \& Distribution, Cleveland 29.18-20, May 1988.

089. "WHAT'S new is auto ID". Distribution, Radnor, 87:104+, Sept. 1988.

\section{7}

090. ABEND, J. "Tracking UPC growth". Stores, New York, 69.52+, Sept. 1987.

091. "UPC + QR = JIT inventory replenishment". Stores, New York, 69.44-54, May 1987.

092. *ACHABAL, D.D. \& McINTYRE, S.H. "Information technology is reshaping retailing". Journal of Retailing, New York, 63(4):321-5, Winter 1987.

093. *AQUINO, Miriam de "Automação comercial sai da incubadeira". Dados \& Idéias, São Paulo, 12(113):14-18, out. 1987.

094.ASSOCIAÇÃO BRASILEIRA DE AUTOMAÇÃO COMER-
CIAL. Manual do código de barras. São Paulo, 1987. 92p.

095. DEIRLEIN, R. "Bar coding and maintenance". Beverage World, Great Neck, 106:76, Feb. 1987.

096. “DIRECTORY assistance: bar coding". Distribution, Radnor, 86: 50-1, Jan. 1987

097. DONOHUE, J.F. "Bar code readers check out as most perplexing automation tool". $M i$ ni-Micro Systems, Denver, 20:4650, June 1987

098. ELLENBOGEN, M. "Distributor bar codes his customers". Industrial Distribution, Radnor, 76:152+, May 1987.

099. ETTORRE, J.J. "Auto ID takes off in transport". Transportation \& Distribution, Cleveland, 28:22-4, Oct. 1987.

100. EVANS, S. \& GRAND, D. "A code of a different stripe". Security Management, Arlington, 31:32-5+, Mar. 1987.

101. "FILE tracking: bar codes improve mortgage service". $A B A$ Banking Journal, New York, 79.28, Sept. 1987.

102. FODOR, G. "Bar code terminology". Industrial Distribution, Radnor, 76:67, Nov. 1987.

103. "Encode, label and scan your way to productivity". Industrial Distribution, Radnor, 76:61+, Nov. 1987.

104. GALVIN, A. "Coding enters the laser age (or does it?)". Beverage World, Great Neck, 106:110+, June 1987.

105. GOLDBERG, G. \& GOLDBERG, J. "The progress of Bookland EAN". Publishers Weekly, Marion, 232:51-5, Oct. 161987

106. "HEALTH care industry moves to use of bar codes". Employee Benefit Plan Review, Chicago, 42:70+, Oct. 1987.

107. "HOW Morse Shoe sees UPC evolving". Stores, New York, 69.55, Mar. 1987.

108. "INFORMATION system puts dyehouse on accurate track". Textile World, Chicago, 137:91, Aug. 1987.
109. "LA CHOY goes real time with tracking". Transportation \& Distribution, Cleveland, 28:72+, Sept. 1987

110. MCDONALD, R. "Bar code: a new data language". Computers \& People, Newtonville, $36(1 / 2): 23-6$, Jan./Feb. 1987.

111. MUELLER, E.J. "The computer challenge: automatic ID". Distribution, Radnor, 86:57+, Aug. 1987.

112. "What should I put in my code? (for physical distribution)" Distribution, Radnor, 86:64, Aug. 1987.

113. "What type of hardware will I need?" Distribution, Radnor, 86:62, Aug. 1987.

114. *PAIVA, Jenner de. "Código de Barras: uma pedra no caminho da automação comercial". Telebrasil, Rio de Janeiro, $9(5): 44-5$, set./out. 1987.

115. PAYNE, N. "Bar code indexing: automating input to document management systems". Administrative Management, New York, 48:31, Oct. 1987.

116. RAYNER, B.C.P. "Bar coding: scanning a $\$ 1$ billion business". Electronic Business, Denver, 13:36-8+, July 151987.

117. "A REVOLUÇÃO do código de barras". Meio \& Mensagem, São Paulo, 278:14, 12 out. 1987.

118. "SCAN-tech 87 to put auto I.D. into focus (Kansas City)". Industrial Distribution, Denver, 76:49, Aug. 1987

119. "SPECIAL section: automatic identification is flying high". Industry Week, Cleveland, 234:A2-A44, Aug. 241987

120. SPIZZIRI, M. "Bar code system earns its stripes (Fisher Corp's use of bar coding equipment in their shipping-order entry system)". Traffic Management, Denver, 26:88-9, Apr. 1987.

121. *STEC, S. "What are some of the applications of bar code technology?" Management Accounting, Montvale, 69(1):53, July 1987.
122. "SUPER scan (automatic identification suppliers guide)". Transportation \& Distribution, Cleveland, 28:27-8+, Oct. 1987.

123. THOMAS, C.M. "Bar coding helps GM on inventory". Automotive News, Detroit:56, Aug. 3 1987.

124. "USPS urges bar code use". Direct Marketing, Garden City, 50:88, Dec. 1987.

125. WEINSTEIN, S. "Pat Collins of Ralphs". Progressive Grocer, Stamford, 66:99-100, June 1987.

126. ZURIER, S. "Bar coding slashes order processing time 88\%". Industrial Distribution, Denver, 76:52-3+, July 1987

\section{6}

127. "AUTOMATIC I.D. family tree sprouts roots". Handling \& Shipping Management, Cleveland, 27:39-50, 0ct. 1986.

128. "AUTOMATIC identification suppliers guide". Handling \& Shipping Management, Cleveland, 27:40-50, Oct. 1986.

129. "BAR-code shipping system cuts labor costs by $\$ 80,000$ ". Traffic Management, Denver, 25:81-3, Jan. 1986.

130. "BAR-code technology comes to check processing arena (Republic Bank, Dallas)". $A B A$ Banking Journal, New York, 78:38+, Mar. 1986.

131. "BAR codes", Automotive News, Detroit:D24, Mar. 24 1986.

132. "BAR codes move from supermart to factory". Purchasing, Denver, 101:26, Sept. 11 1986.

133. "BAR coding: an important link". Industry Week, Cleveland, 229. 90, May 261986.

134. "BAR coding: medium for Quinck Response". Textile World, Chicago, 136:56-7, Dec. 1986.

135. "BAR coding helps separator attend to job details (Viking Graphics, Chicago)". American Printer, Chicago, 196.64+, Mar.1986. 
136. BOLLE, S. "The book industry moves toward a bar code standard". Publishers Weekly, Marion, 229.77-9, May 231986.

137. BRASIL. MINISTÉRIO DA INDÚSTRIA E DO COMÉRCIO. CONSELHO DE DESENVOLVIMENTO COMERCIAL. COORDENADORIA DE EXTENSÃO COMERCIAL. Automação comerciat. código nacional de produtos. Brasília, 1986. 24p. (Cadernos Técnicos, 25)

138. BROOKE, L. “Non-visible bar code system unveiled". Automotive Industries, Radnor, 166:105, May 1986.

139. CALLARI, J.J. "Bar codes: a perfect fit for Levi Strauss". Traffic Management, Denver, 25:75+, Nov. 1986.

140. *COCHRAN, J. "Bar-code scanning for applications for patents (US Patent Office)". Computers and People, Newtonville, 35(3/4):12-15, Mar./Apr. 1986.

141. "CÓDIGOS de barras". Elektor Eletrônica, São Paulo, 1(4):29-33, out. 1986

142. COOKE, J.A. "Bar-code software lags behing hardware". Traffic Management, Denver, 25:17+, Dec. 1986.

143. COOKE, J. "New barcode scanner fights pirate distribution". Traffic Management, Denver, 25:20, Feb. 1986.

144. GANZEL JR., N. "Bar code data entry (U.S. Army)". American Shipper, Jacksonville, 28:6+, Feb. 1986.

145. HENRIQUES, D. "Growth in line: Intermec cashes in on expanding market". Barron's, Chicopee, 66:14+, July 211986.

146. LEÃO, Manoel Luiz. "Aplicação do código de barras na indústria". Máquinas e Metais, São Paulo, 22(249):12-22, ago. 1986.

147. "A LEITURA dos códigos de barras". Elektor Eletrônica, São Paulo, 1(5):25-32, nov. 1986.

148. MOCIULEWSKI, J. "Bar code use today". Distribution,
Radnor, 85: 10-12, Mar. 1986. (supp.)

149. MUSTARDO, A.S. \& JOHNSTON, C. "Bar-code bonanza: labeling equipment smooths existing jobs at a social services association". Graphic Arts Monthly and the Printing Industry, New York, 58:40, June 1986.

150. "ON-SITE bar code equipment (paper mills; table)". Paper Trade Journal, Lincolnshire, 170.43, May 1986.

151. "SCAN-tech raging success; scan-two due in April (Baltimore)". Handling \& Shipping Management, Cleveland, 27:1718, Jan. 1986.

152. "SCAN-tech shows grown-up auto ID (San Francisco)". Handling \& Shipping Management, Cleveland, 27:23-4+, Dec. 1986.

153. SCHULZ, D. "Is UPC in your future?" Stores, New York, 68:36-42, Sept. 1986.

154. *SLOAN, P. \& DAGNOLI, J. "UPC registers retailing impact". Advertising Age, Chicago, 57(25):3+, Apr. 71986.

155. WINTER, D. "Bar codes win: easier tracing of parts trims GM's recalls". Ward's Auto World, Detroit, 22:66, Jan. 1986.

\section{5}

156. "AUTOMATED identification suppliers guide". Handling \& Shipping Management, Cleveland, 26:40+, Nov. 1985.

157. BAKER, Eugene F. Industry shows its stripes: a new role for bar coding. New York, AMA, 1985. 85p.

158. "BAR coding". Handling \& Shipping Management, Cleveland, 26:92, Jan. 1985.

159. "BAR-coding-textiles takes a closer look (in textile mills)". Textile World, Atlanta, 135:40-1, Nov. 1985.

160. BELLI, C. "It's a bar code world (Intermec's D.C. Allais)". Automotive News, Detroit:D6, Oct. 71985.

161. "BUILDING a better coupon (universal product code)". Progressive Grocer, Stamford, 64:60, May 1985

162. BUREAU, W.H. "Electronic paper management (Embarc)". Graphic Arts Monthly and the Printing Industry, New York, 57:62+, Aug. 1985.

163. CALLAS, J. "Bar-coding systems: how bars and spaces talk to computers". Traffic Management, Denver, 24:121, Aug. 1985.

164. CIANCI, W. "Implementing bar coding systems improves data flow and productivity (paper manufacturing operation)". Pulp \& Paper, San Francisco, 59.7983, June 1985.

165. COCHRAN, John J. "Application of on-line bar-code scanning use for management and control of the patent application process". In: AIM Proceedings. Papers... Baltimore, 1985. p.34-9.

166. "COMPUTER printing system handles bar coding/graphics applications (Emblem's System 1800)". Graphic Arts Monthly and the Printing Industry, New York, 57:S74, July 1985

167. "CUTTERS to mills: how to improve service (between textile and apparel companies)". Textile World, Atlanta, 135:46+, Nov. 1985.

168. HARMON, C.K. "Help in evaluating bar code equipment". Handling \& Shipping Management, Cleveland, 26:11, Apr. 1985

169. HARMON, C.R. "Standardizing bar codes". Handling \& Shipping Management, Cleveland, 26:9, Mar. 1985

170. "INDEX of automated identification suppliers". Handling \& Shipping Management, Cleveland, 26:48+, Nov. 1985.

171. "IS bar coding right for you?" Traffic Management, Denver, 24: 89+, Apr. 1985.

172. JOHNSON, M. "UPC update: shaping up the simbol (supermarkets)". Progressive Grocer, Stamford, 64:93-4+, Mar. 1985

173. "NRMA urges dual technology - OCR-A, UPC - for UVM".
Stores, New York, 67:51, Oct. 1985.

174. "THE PICKS of Pro Mat ' 85: lift trucks, labels, automation". Purchasing, Denver, 98(10):99+, May 231985.

175. "PUTTING bar code to the test". Handling \& Shipping Management, Cleveland, 26:32, Nov. 1985.

176. SOBCZAK, Thomas $V$. "Machine-readable marking codes: who specifies what type of symbology?" In: ___ Applying industrial bar coding. Michigan, Society of Manufacturing Engineers, 1985. p.17-20.

177. "TALKING in bar code (data acquisition technology)". Handling \& Shipping Management, Cleveland, 26.39, Nov. 1985.

178. TERESKO, J. "Bar coding: why the boom?" Industry Week, Cleveland, 224:63-4, Feb. 181985.

179. THOMAS, P. "Stamping out the paper glut with bar codes (In Focel's Paper Flo system)". Venture, New York, 7:110, Sept. 1985.

180. "UVM ticketing campaign gathering vendor momentum (Universal Vendor Marking)". Chain Store Age Executive with Shopping Center Age, New York, 61:95-6, July 1985.

181. VAN DER TUUK, T. "Keep it simple to get the most out of bar codes". American Printer, Chicago, 194:71-2, Mar. 1985.

182. "WILL department stores be next UPC stronghold? (Universal Product Code)". Chain Store Age Executive with Shopping Center Age, New York, 61:64, Dec. 1985.

183. WINTER, D. "Belly up to the bar code (autoparts)". Ward's Auto World, Detroit, 21:45, Aug. 1985.

184. "WRAY, B.R. "Bar codes: basic principles". Computers and People, Newtonville, 34(1/2):1215, Jan./Feb. 1985.

\section{4}

185. "BAR codes: from cans to cars". Automotive News, De- 
troit:D6, June 181984.

186. "BAR coding gains in manufacturing segment". Handling \& Shipping Management, Cleveland, 25:22+, Feb. 1984.

187. BROWN, C.J. "JIS system can aid trade (Japanese Industrial Standards Marking System)". Iron Age Metals Producer Radnor, 227: 25, June 181984.

188. CALLAHAN, J.M. "Automotive bar codes take off". Automotive Industries, Detroit, 164:57-9, June 1984

189. "THE CODEMAKERS" Forbes, New York, 134:12-3, July 301984

190. "CODES for carmakers". Forbes, New York, 134(15):12-3 Dec. 311984.

191. "ENTERING the bar code age". American Printer, Chicago, 193: 170+, Sept. 1984.

192. *FRANCESCHINI, R. D. "Bar coding: an important technology comes of age". Purchasing, Denver, 96(12):107+, June 211984.

193. HIGGINS, K. "UPC codes represent newest method of combating coupon misredemption". Marketing News, Chicago, 18:15, Sept. 281984.

194. "INDEX-suppliers of bar code products and services". Handling \& Shipping Management, Cleveland, 25:56+, Nov. 1984.

195. "JOURDAN goes UPC one better (C. Jourdan)". Chain Store Age Executive with Shopping Center Age, New York, 60(sec. 2):4+, May 1984.

196. "LABELS, labels, labels (bar code labeling system, of U.S. Dept. of Agriculture's National Finance Center)". Modern Office Technology, Cleveland, 29.78+, July 1984 .

197. "MAKE the commodity code a real tool". Purchasing, Denver, $96(12): 93+$, June 21 1984.

198. "OCR-A-because it's not peas (bar-code standards pro- gram)". Chain Store Age Executive with Shopping Center Age, New York, 60 (sec. 2):7, May 1984

199. PARSONS, K. \& MAGINNIS, C. "Verification: key to effective bar coding". Handling \& Shipping Management, Cleveland, 25:38-40+, Nov. 1984.

200. "QUICK selection guide bar coding products, services and suppliers (table)". Handling \& Shipping Management, Cleveland, 25:50+, Nov. 1984.

201. QUINLAN, J. "Getting into bar coding". Handling \& Shipping Management, Cleveland, 25:49-54, Nov. 1984

202. ROZEN, M. "Barcoding moves to the factory floor". Dun's Business Month, New York 124:77, Aug. 1984.

203. TRUNICK, P.A."Outlook'84 labeling, marking, bar coding". Handling \& Shipping Management, Cleveland, 25:75-6, Jan. 1984.

204. "WELCOME to open-date coding". Beverage World, Great Neck, 103: 66+, June 1984.

\section{3}

205. "BAR CODES: crossing the Atlantic". The Economist, London, 286(7275):87, Feb. 5 1983.

206. BARRET, G.S. "Bar codes: new weapon in paper inventory arsenal". American Printer, Chicago, 191:38+, Sept. 1983.

207. *BROWN, P.B. "If at first you don't succeed (Symbol technologies, Inc. deals solely in the business of bar codes)". Forbes, New York, 132(5):74, Aug. 29 1983.

208. DENSFORD, L.E. "Legal cloud lifts on coupon scanning (Kaslow's controversial patent, UPC symbols on coupons)". Progressive Grocer, Stamford, 62:15, Nov. 1983

209. *HOLOGRAMS: working pictures (holographic optical elements)". The Economist, London, 286 (7281):100-1, Mar. 191983.
210. "INDUSTRY steps up to the bar code". S \& MM, New York, 130.14, May 161983.

211. "PACKAGING, marking and labeling: bar code demand just beginning". Traffic Management, Denver, 22:52-3, Apr. 1983.

212. SANGER, E. "No magic wands; a scan of the hand-held laser scene". Barrons, Chicopee, 63:15+, Apr. 41983.

213. "SCANNING grows as means of tracking sales patterns". Quick Frozen Foods, Duluth, 45:46-7, Mar. 1983.

214. "SOLVING POS puzzles: which code? which entry mode?" Stores, New York, 65:52-4, Mar. 1983.

215. *TARSIA, F. "Bar codes control stock transfers (bar code technology records and captures data for an envelope control system at the National Securities Clearing Corporation)". Journal of Systems Management, Cleveland, 34(10):22-3, Oct. 1983

216. TRUNICK, P.A. "The move to bar codes". Handling \& Shipping Management, Cleveland, 24:30-2+, Nov. 1983.

217. "USE scanner data analysis to avoid pitfalls in marketing models". Marketing News, Chicago, 17(sec.2):16+, Jan. 211983.

218. WESTERMAN, M. "Electronic coder meets test". Beverage World, Great Neck, 102:66, Mar. 1983.

\section{2}

219. "AUTO group proposes bar code standards". Iron Age, Radnor, 225: 17, July 51982.

220. "BAR code picked for 'greater flexibility' “. Infosystems, Wheaton, 29(3)pt2:13, Mar. 1982.

221. CATALANO, F. "Bar codes: the industrial solution to shop-floor data entry". Mini-Micro Systems, Boston, 15:155-6+, Sept. 1982.

222. GANZ, J.M. "The UPC goes military". Security Manage- ment, Arlington, 26:108+, Sept. 1982.

223. "GOVERNMENT expands bar code use". Industrial Marketing, Chicago, 67:16-17, Oct 1982.

224. "NEW codes promise more usable sales figures". Progressive Grocer, Stamford, $61: 52-3$, July 1982

225. NULTY, P. "The bar-coding of America". Fortune, New York, 106: 98-101, Dec. 271982.

226. PHILLIPS, N.I. "Bar codes could be an automotive magic wand". Automotive News, Detroit:E22, July 51982

\section{1}

227. *'DISTRIBUTORS push uniform numbers". Purchasing Denver, 90. 18C6, Feb. 261981.

228. "LATROBE Brewery cuts coding cost $50 \% "$. Beverage Industry, New York, 71:108-9, Nov. 201981 .

229. LIPTON, J.M. "OCR-A undate". Stores, New York, 63:46-8, July 1981 .

230. *MILLER, F.W. "Magic wand comes of age with new uses". Infosystems, Wheaton, 28(7):42+, July 1981

231. MOSKAL, B.S. "Breakthrough in material handling". Industry Week, Cleveland, 208:912, Jan. 261981.

\section{9}

232. "BAR code system cuts re-order time in half". Infosystems, Wheaton, 26(7):54, July 1979.

\section{7}

233. BIBLIOTECA NACIONAL. AGÊNCIA BRASILEIRA DO ISBN. Sistema ISBN. Rio de Janeiro, Biblioteca Nacional/CIMEC/SNEL, 1977.7p.

\section{SEM DATA}

234. BENEDETTI, J. Código de barras. tipos e aplicações. São PauIo, s.c.p., s.d., 19p. (separata). $\square$ 